$14^{\text {th }}$ International Conference on

AEROSPACE SCIENCES \& AVIATION TECHNOLOGY,

ASAT - 14 - May 24 - 26, 2011, Email: asat@ mtc.edu.eg

Military Technical College, Kobry Elkobbah, Cairo, Egypt

Tel: +(202) 24025292-24036138, Fax: +(202) 22621908

\title{
Interference Cancellation with RZF Equalization for the Downlink MC-CDMA Systems
}

\author{
B.A. Al-fuhaidi ${ }^{*}$, H.E.A. Hassan ${ }^{\dagger}$, S.S. Alagooz ${ }^{\ddagger}$, M.M. Salah ${ }^{\S}$,
}

\begin{abstract}
The Multi-Carrier Code Division Multiple Access (MC-CDMA) is becoming a very attractive multiple access technique for high-rate data transmission in the future wireless communication systems. The advantage of MC-CDMA systems that is work in frequency domain. Recently, MC-CDMA with any equalization scheme has attracted much attention for its ability to obtain an excellent performance even in strong frequency selective fading channels. In this paper, the two proposed architecture of the downlink MC-CDMA is based on the regularized zero forcing equalizer (RZF) with parallel interference cancellation (PIC) RZF-PIC) and the rake receiver with parallel interference cancellation (PIC) (RAKE-PIC) to suffer from the intersymbol interference (ISI) and multiple access interference (MAI). Two schemes are implemented in frequency domain. The effects of the tentative decision functions, the user loading on the performance of the proposed receivers architecture, and the number of cancelled users are discussed in this paper. Simulation results show the advantage of the RZF equalizer result over rake receiver and show that the combination of PIC and equalization provides an efficient solution to suppress the MAI and ISI in downlink MCCDMA systems over frequency selective fading channels.
\end{abstract}

Keywords: Downlink MC-CDMA, Decision functions, RAKE-PIC, ZF, and RZF equalizer.

\section{Notations}

The symbols $(.)^{H},(.)^{T}$, and $(.)^{-1}$ designate complex conjugate transposition, transposition of a matrix, and the inverse of a matrix, respectively. Vectors and matrices are represented in boldface. $F^{-1}$ and $F$ denote the inverse fast Fourier transform and the fast Fourier transform operators, respectively.

\section{Introduction}

Multi-carrier code-division multiple-access (MC-CDMA) with advantages of simple channel equalization and robustness against multipath distortion has recently received considerable attention in wireless communication systems [1]. The multi-carrier CDMA, the combination of the orthogonal frequency division multiplexing (OFDM) and CDMA systems, is one of the attractive techniques for future wireless communication systems to provide high level of user traffic along a high-quality service. It can be used to overcome the capacity limit of the conventional DS-CDMA system. The benefits of MC-CDMA include high spectral

\footnotetext{
*Yemeni Armed Forces, belalarh@gmail.com

†Egyptian Armed Forces, hossameldin_aboubakr@hotmail.com $\ddagger$

${ }^{\S}$ Egyptian Armed Forces,
} 
efficiency, easy adaptation to severe channel conditions without complex detection, and robustness against intersymbol interference (ISI) and fading caused by multipath propagation [3]. There are several variations of MC-CDMA, such as multicarrier direct-sequence CDMA (MC-DS-CDMA) and multitone CDMA [3]. These signals can be easily transmitted and received using the fast Fourier transform (FFT) without increasing the transmitter and receiver complexities, and have the attractive feature of high spectral efficiency [2,3].MAI, ICI and the ISI will down the performance of the multi user communication system that reduce the transmitted power required to meet the target signal-to noise ratio (SNR), and enable more channels to be transmitted with a limited power.

Various receiver architectures have been proposed to combat the MAI, the ICI, and the ISI, such that the RAKE receiver that is commonly used for the detection of transmitted symbols in CDMA systems, and frequency domain equalization such that zero forcing, MMSE and regularized zero forcing equalizer $[4,5]$. Frequency domain equalization is reduce the complexity when compared to the time domain equalizer and the rake receiver.

The insertion of the cyclic prefix is reducing the ISI. And the equalization can still not prevent from the MAI. Hence, it is necessary to employ multiuser detection to improve the performance. There are other block transmission schemes for MC-CDMA such as MCCDMA with known symbol padding and MC-CDMA with zero padding [6, 7, 8]. The joint use of nonlinear FDE and MAI cancellation for the DS-CDMA uplink has been considered [14]. In [15], PIC and FDE are used to enhance the performance of the downlink zero padding CDMA systems. The Performance evaluation of cyclic prefix CDMA systems with frequency domain interference cancellation is studied in [16].

In this paper the performance of the downlink CP-MC-CDMA is enhancement by eliminating or mitigating MAI and ISI. A hybrid algorithm of detection comprising both linear equalization and tentative decision functions with PIC in MC-CDMA is suggested and studied in the paper. Two efficient interference cancellation architectures are proposed to be used in this hybrid algorithm. Such that the RAKE-PIC and RZF-PIC algorithms. In these algorithms the MAI is regenerated and subtracted from the received signal in the frequency domain before equalization. The effective of the tentative decision (hard, tanh, null zone, unit clipper and soft) functions on the two proposed receivers are studied in this paper and show the advantage of any decision function to each other. The comparison between these algorithms are studied in this paper to show the enhancement performance of the adding regularized parameter to the zero forcing equalizer rather than the rake receiver and zero forcing equalizer.

The paper is organized as follows. In Section 2, the system model for the downlink MCCDMA communication system is presented. In Section 3 the proposed two frequency domain interference cancellation structure are presented. In section 4 the different decision functions are presented. Computer simulations for several situations to demonstrate the performance of the system and conclusions are given in Sections 5 and 6, respectively.

\section{System Model}

The downlink MC-CDMA block transmission with K active users over a frequency selective fading channel is considered. The downlink transceiver structure of the proposed scheme is presented in Fig. 1. An uncoded synchronous binary phase-shift keying (BPSK) MC-CDMA system with $K$ users, $N$ chips per symbol, those symbols are spread using a certain spreading code. After spreading, the resulting signal is scrambled using a complex scrambling sequence, after that the inverse fast Fourier transform (IFFT) applied to the resulting signal and a cyclic prefix of $\mathrm{N}_{\mathrm{CP}}$ chips is added at the beginning of each block to prevent possible ISI in MCCDMA systems ) which contains a copy of the last part of the MC-CDMA symbol. The length 
of the cyclic prefix must be greater than the maximum excess delay of the channel to accommodate for the interblock interference (IBI). The transmitted signal is then passed a frequency selective multipath fading channel with additive white Gaussian noise (AWGN). At the receiver, the cyclic prefix is removed and the received symbols are sent to an FFT block to demultiplex the multicarrier signal.

The baseband channel response can, then, be expressed as follows [9,13]:

$h(t)=\sum_{l=0}^{L-1} h_{1} \delta\left(t-\tau_{1}\right)$,

where $h_{1}$ and $\tau_{1}$ represent the complex fading and the propagation delay of the lth path, and $\mathrm{L}$ is the number of multipath components of the channel impulse response $h$. In this paper, we assume block fading, where the path gains stay constant over each block duration.

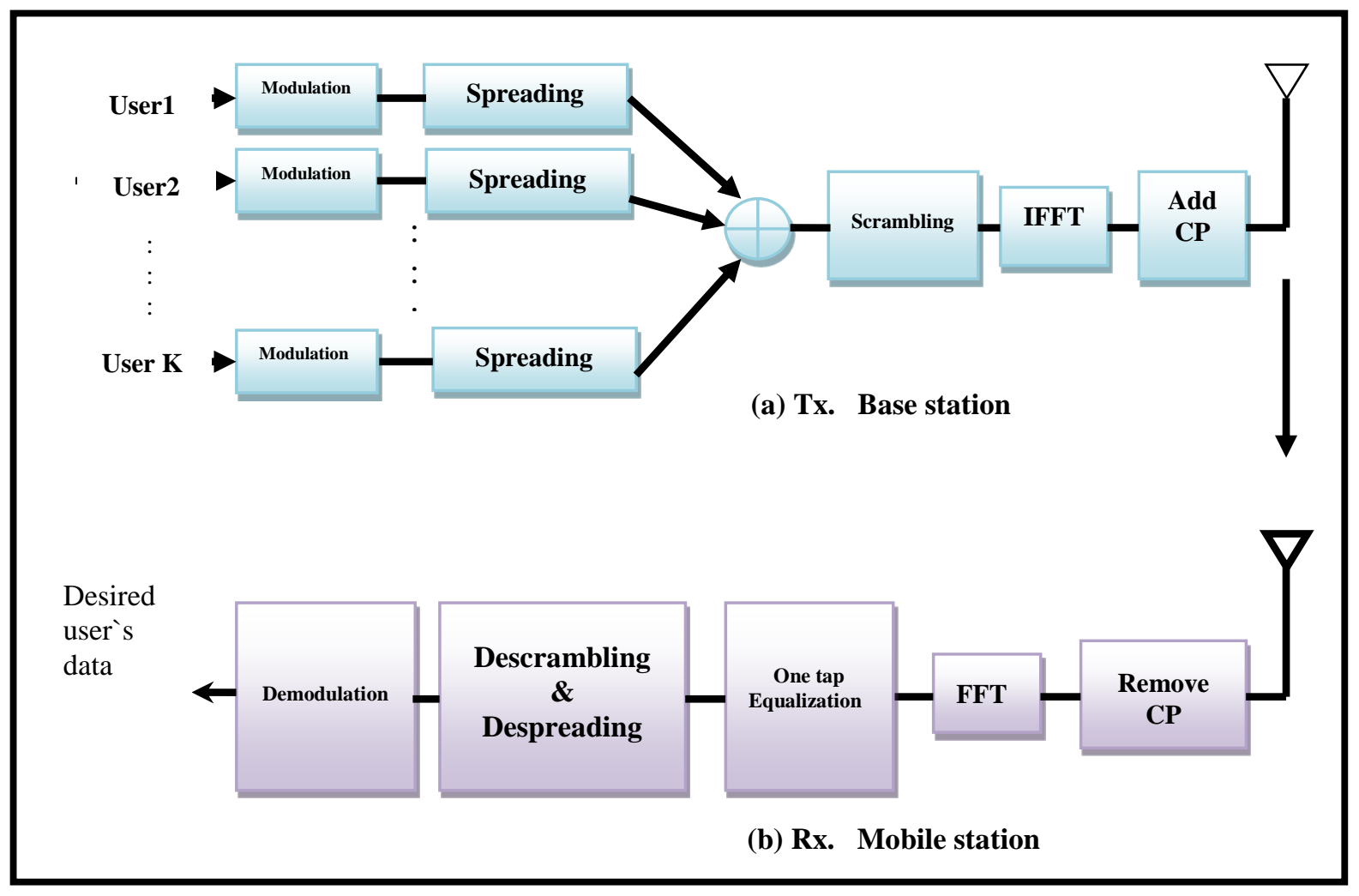

Fig. 1 Downlink MC-CDMA

The received block after removal of cyclic prefix can be formulated as:

$\mathbf{r}=\mathbf{H}_{\mathrm{C}} \mathbf{d}+\mathbf{n}$,

where $\mathbf{d}$ is an $\mathrm{NM} \times 1$ vector representing the block of the transmitted chip sequence, $\mathbf{r}$ is the received vector, $\mathbf{n}$ is the additive noise, and $\mathbf{H}_{\mathrm{C}}$ is an $\mathrm{NM} \times \mathrm{NM}$ circulant matrix describing the multipath channel. $\mathbf{H}_{\mathrm{C}}$ can be written as follows:

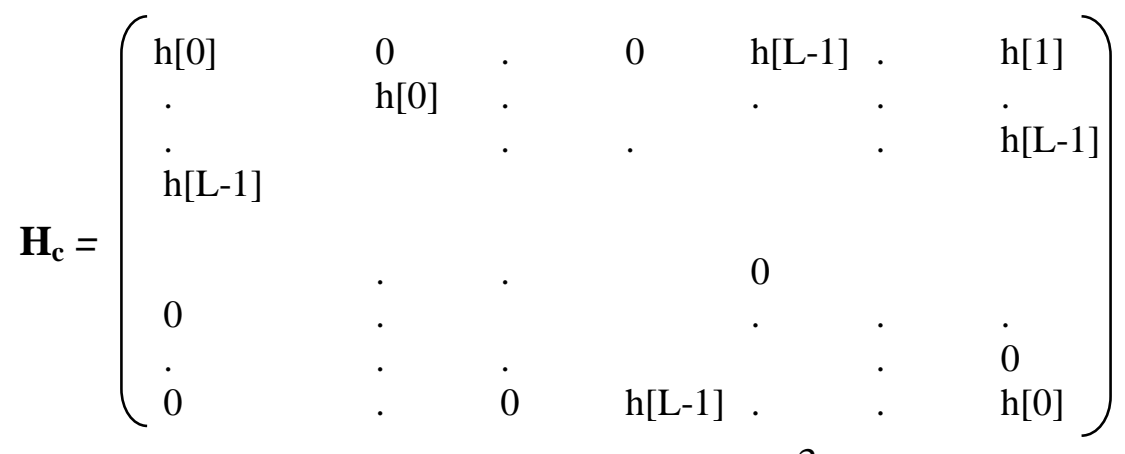


The vector $\mathbf{d}$ can be represented as:

$$
\mathbf{d}=\mathbf{F}^{-1} \mathbf{C S b}
$$

where $\mathbf{F}^{\mathbf{- 1}}$ is an $\mathrm{NM} \times \mathrm{NM}$ Inverse Fast Fourier transform, $\mathbf{C}$ is an $\mathrm{NM} \times \mathrm{NM}$ scrambling code matrix, $\mathbf{S}$ is an $\mathrm{NM} \times \mathrm{KM}$ block diagonal matrix whose diagonals contain the spreading vectors, and $\mathbf{b}$ is a $\mathrm{KM} \times 1$ vector consisting of the users' data. The structures of the individual components in Eq. (4) are given bellow [12]:

$$
\begin{aligned}
& S=\operatorname{diag}[\bar{S}, \bar{S}, \ldots, \bar{S}], \\
& \bar{S}=\left[s_{1}, s_{2}, \ldots, s_{K}\right], \\
& s_{K}=\left[s_{K}(\mathbf{0}), s_{K}(1), \ldots, s_{K}(N-1)\right]^{T}, \\
& \mathbf{b}=\left[\mathbf{b}^{\mathrm{T}}(\mathbf{1}), \mathbf{b}^{\mathrm{T}}(2), \ldots, \mathbf{b}^{\mathrm{T}}(\mathbf{M})\right], \\
& \mathrm{C}=\operatorname{diag}[\mathbf{c}(\mathbf{1}), \mathbf{c}(2), \ldots, \mathbf{c}(\mathbf{N M})], \\
& \mathbf{b}(\mathbf{m})=\left[\mathrm{A}_{1} \mathbf{b}_{1}(\mathbf{m}), \mathrm{A}_{2} \mathbf{b}_{2}(\mathbf{m}), \ldots, \mathrm{A}_{\mathrm{K}} \mathbf{b}_{\mathrm{K}}(\mathbf{m})\right]^{\mathrm{T}},
\end{aligned}
$$

where $\mathbf{s}_{\mathrm{K}}$ is the spreading code of the kth user. Equation (2) can be written in terms of the MAI as follows:

$$
\mathbf{r}=\mathbf{H}_{\mathbf{C}} \mathbf{F}^{-1} \mathbf{C S}_{\text {des } \mathbf{b}_{\text {des }}}+\mathbf{H}_{\mathbf{C}} \mathbf{F}^{-1} \mathbf{C U b}_{\text {int }}+\mathbf{n}=\underbrace{\mathbf{H}_{\mathbf{C}} \mathbf{d}_{\text {des }}}_{\text {Useful diversity }}+\underbrace{\mathbf{H}_{\mathbf{C}} \mathbf{d}_{\text {int }}}_{\text {MAI }}+\underbrace{\mathbf{n}_{\text {w }}}_{\text {Noise }},
$$

where $\mathbf{b}_{\text {des }}$ is an $M \times 1$ vector consisting of the desired user's bits, $\mathbf{b}_{\text {int }}$ is a $(K-1) M \times 1$ vector consisting of the interfering users' bits, $\mathbf{S}_{\mathrm{des}}$ is an $N M \times M$ matrix consisting of the spreading code of the desired user, and $\mathbf{U}$ is an $N M \times(K-1) M$ matrix consisting of the spreading codes of the interfering users.

From Eq. (11), it is found that only the first term contains the desired data, the second term is due to the MAI, and the third term is a noise term.

\section{Interference Cancellation for Downlink MC-CDMA}

\subsection{RAKE Receiver with PIC}

This scheme uses the RAKE receiver and PIC to estimate, regenerate, and cancel all the interfering users. Then, the RAKE receiver is used to provide the enhanced desired user's data. This scheme is called (RAKE-PIC). It is shown in Fig. 2. The steps of the RAKE-PIC algorithm can be summarized as follows:

- After the received signal transferred from the base station the cyclic prefix is removed from the received signal.

- After removing the cyclic prefix, the FFT is applied to the received signal, With the aid of Eq. (11), we get:

$R_{T}=E D_{\text {des }}+E D_{\text {int. }}+\mathbf{N}$

where $\boldsymbol{E}$ is an equalization diagonal matrix and $\mathbf{D}_{\text {des }}, \mathbf{D}_{\text {int }}$ and $\mathbf{N}$ are the Fourier transforms of $\mathbf{d}_{\text {des }}, \mathbf{d}_{\text {int }}$, and $\mathbf{n}$, respectively. 
- The resulting signal after the FFT is first sent to the frequency domain channel estimator which estimates the channel coefficients.

- The estimate of the channel coefficients is used at stage one to estimate the symbols of the interfering users with RAKE receiver. The estimates of the symbols obtained here are the first decision made, and we refer to this as a tentative decision. This step can be written as follows:

$\hat{\mathbf{b}}_{\text {int }}=\boldsymbol{f}_{\text {dec }}\{\mathbf{U}^{\mathrm{T}} \mathbf{C}^{\mathbf{H}} \underbrace{\mathbf{E}^{\mathrm{H}} \mathbf{R}_{\mathbf{T}}}_{\text {Rake Receiver }}\}$,

where $f_{\text {dec }}($.$) is the tentative decision function (tanh decision function is considered).$

- The estimates of the interfering users' symbols are used with the channel estimate to regenerate the interfering users' signals (MAI) as follows:

$\mathbf{R}_{\text {MAI }}=E F^{-1}\left(C U \hat{b}_{\text {int }}\right)$,

- The MAI is then subtracted from received signal $\mathbf{R}$ to get the frequency domain interference free signal as follows:

$\mathbf{Y}=\mathbf{R}-\mathbf{R}_{\mathbf{M A I}}$,

- A better estimate of the symbols of interest can be obtained by applying the RAKE detection on the interference free signal Yas follows:

$\hat{\mathbf{b}}_{\text {des }}=\boldsymbol{f}_{\operatorname{dec}}\left\{\left(\mathbf{S}_{\mathrm{des}}^{\mathrm{T}} \mathbf{C}^{\mathrm{H}} \mathbf{E}^{\mathrm{H}} \mathbf{Y}\right\}\right.$

where $f_{\text {dec }}($.$) is a hard decision function.$

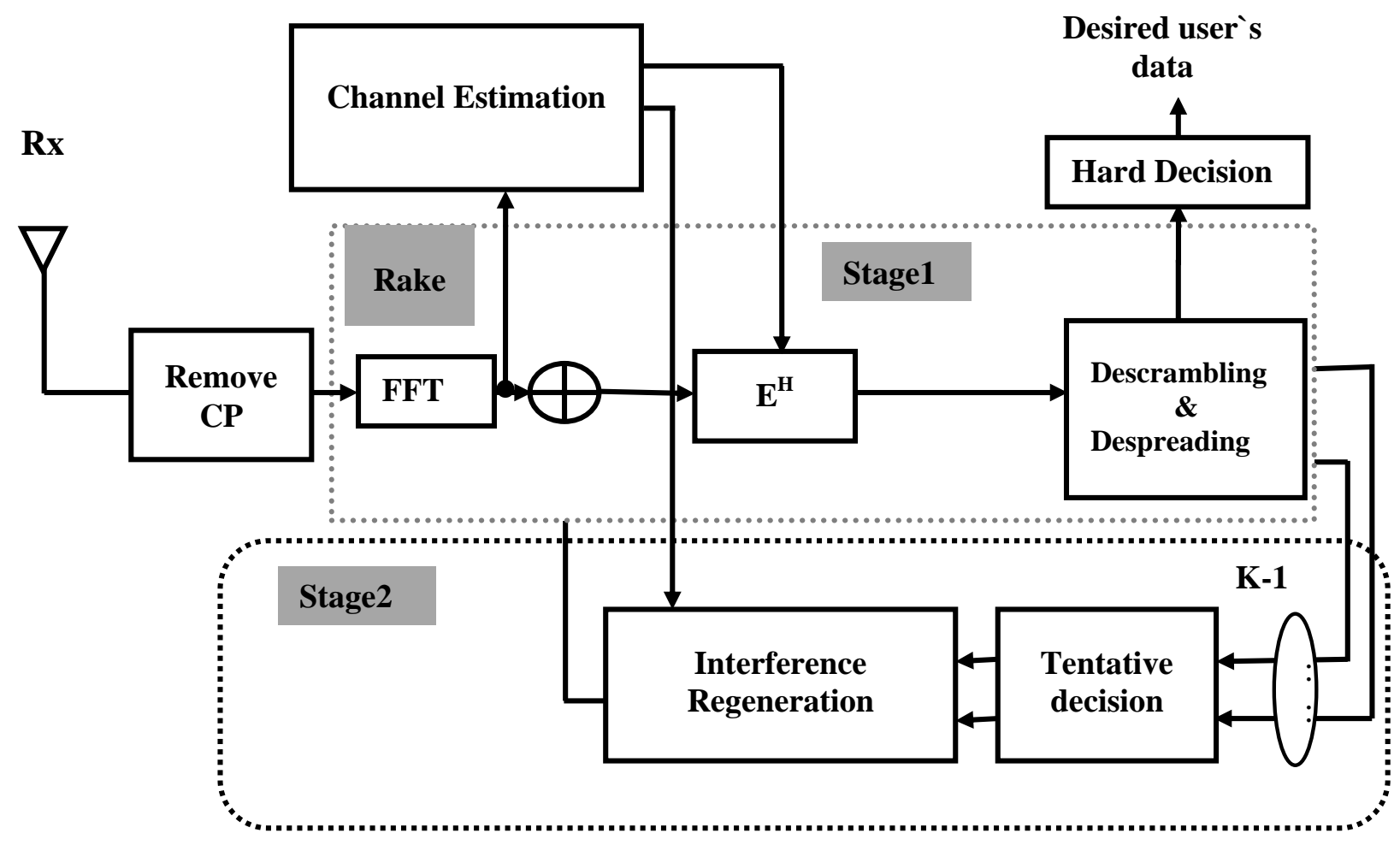

Fig. 2 Structure of the RAKE-PIC for downlink MC-CDMA systems. 
The main advantage of this receiver lies in its low complexity as compared to the other suggested schemes. However, the performance of this receiver deteriorates as the number of users increases. This can be explained by the fact that, for heavy loads, the RAKE receiver sees too much interference which makes its decisions about interfering users unreliable.

\subsection{Equalization RZF with PIC}

This section presents the second suggested scheme which is used to improve the performance of the previous scheme for the downlink MC-CDMA system. The suggested scheme uses an RAKE receiver to estimate the interfering bits. Then, PIC is used to regenerate, and cancel all interfering users' data. After that, RZF equalizer is used to provide a better estimate of the desired user's data. The suggested algorithm is called RZF-PIC (see Fig. 3). The steps of the RZF-PIC algorithm can be summarized as follows:

- After the received signal transferred from the base station the cyclic prefix is removed from the received signal.

- The FFT is applied to this received signal that provided as in Eq. (12).

- After that the channel coefficients are estimated from the resulting signal.

- The symbols of the interfering users are estimated by the RAKE receiver as in Eq. (13).

- The MAI is generated as in Eq. (14).

- The MAI is subtracted from $\mathbf{R}_{\mathrm{T}}$ to get frequency domain interference free signal as in Eq. (15).

- Finally, a better estimate of the symbols of interest can be obtained after RZF equalization, descrambling, and despreading as follows:

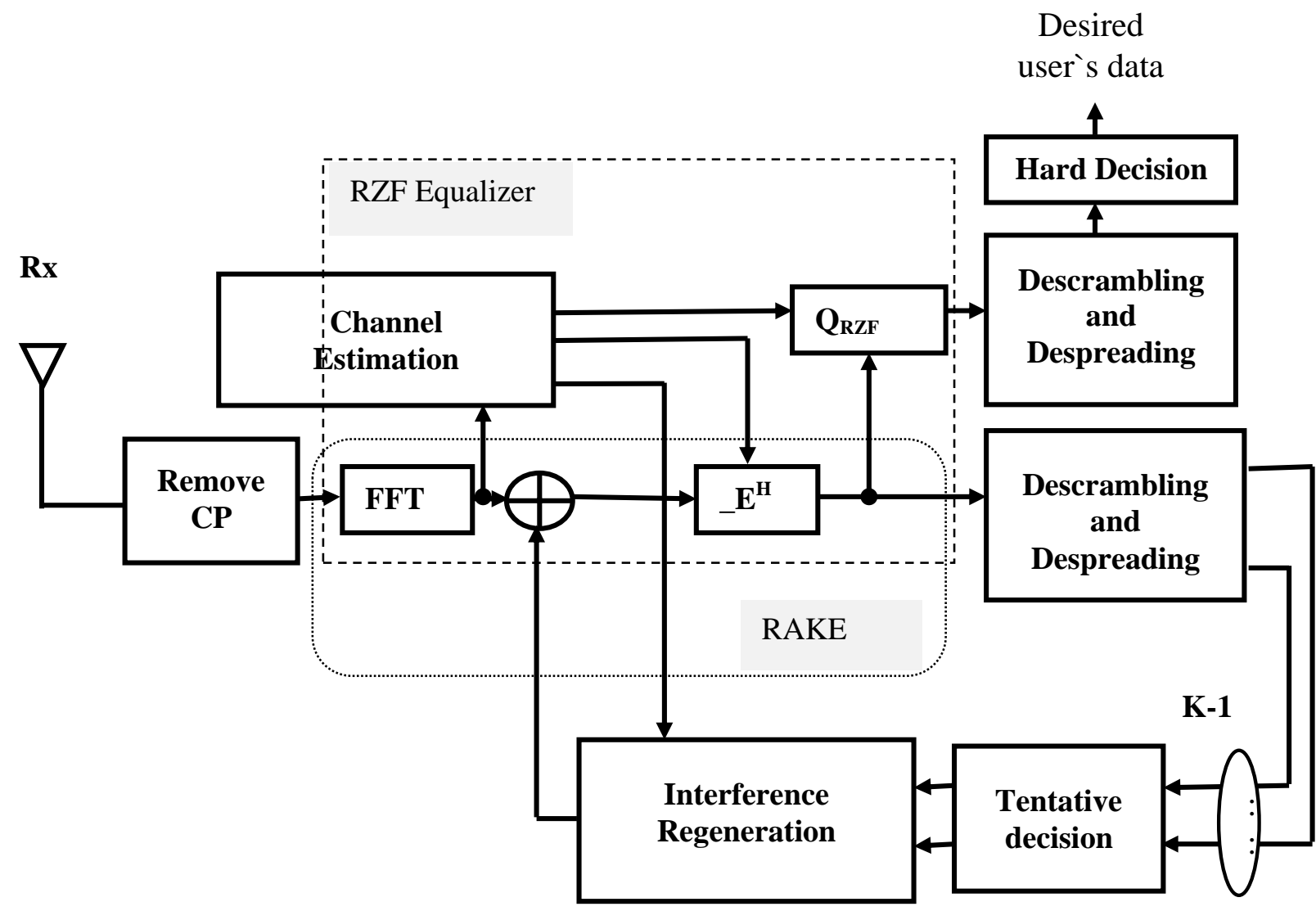

Fig. 3. Structure of the RZF-PIC for Downlink MC-CDMA systems. 


$$
\widehat{\mathbf{b}}_{\text {des }}=f_{\text {dec }}\left\{C_{d e s}^{T} C^{H} W_{R Z F} Y\right\}
$$

The RZF equalization operator is given by:

$$
W_{R Z F}=\left(\mathbf{E}^{H} \mathbf{E}+\alpha \mathbf{I}\right)^{-1} \mathbf{E}^{H}=Q_{R Z F} \mathbf{E}^{H}
$$

It is the regularized zero forcing equalization operator in the downlink MC-CDMA.

The RZF equalizer avoids the noise enhancement caused by the zero forcing equalizer by adding the regularization term $\boldsymbol{\alpha}$. The main advantage of this algorithm is that the statistics of the transmitted data and the additive noise are not required. Its performance is also better than that of the RAKE-PIC algorithm. However, it is more complex than the RAKE-PIC algorithm.

\section{Decision Functions}

The performance of PIC depends on the tentative decision function used. So, due to error propagation, PIC with a hard decision function may perform worse than PIC with linear or soft decision functions [9]. Hard-decision interference cancellation can completely cancel interference only when the hard decisions made are correct which not the case in all decisions is. The most popular decision functions are:

\subsection{The Hard Decision Function}

$y=f_{\text {dec }}(x)=\left\{\begin{array}{r}1, x \geq 0 \\ -1, x<0\end{array}\right.$

It makes a hard decision in the favor of one of the two possible symbols.

\subsection{The Null Zone Decision Function}

$y=f_{\text {dec }}(x)=\left\{\begin{array}{cr}\mathbf{1}, & \mathbf{x}>\mathbf{C}_{\mathbf{n}}, \\ \mathbf{0}, & \mathrm{x} \in\left[-\mathbf{C}_{\mathbf{n}}, \mathbf{C}_{\mathbf{n}}\right], \\ -\mathbf{1}, & \mathrm{x}<-\mathbf{C}_{\mathbf{n}} .\end{array}\right.$

It makes a hard decision when the soft bit estimate lies outside the interval $\left[-C_{n}, C_{n}\right]$, and sets the decision result to zero when the soft bit estimate lies inside this interval. $C_{n}$ is the null zone decision threshold ( $\left.0 \leq C_{n} \leq 1\right)[9,10]$.

\subsection{The Linear Decision Function}

$y=f_{d e c}(x)=x$.

It performs worse than the other decision functions.

\subsection{The Unit Clipper Decision Function}

$y=f_{d e c}(x)=\left\{\begin{array}{rr}\mathbf{1}, & x>1 \\ x & x \in[-\mathbf{1}, \mathbf{1}] \\ -\mathbf{1}, & x<-1\end{array}\right.$

It makes a soft bit decision when the soft bit estimate lies inside the interval $[-1,1]$ to avoid the propagation of errors, and makes a hard decision when the soft bit estimate lies outside the interval $[-1,1]$ to avoid the noise enhancement [11]. 


\subsection{The Tanh Decision Function}

$y=f_{d e c}(x)=\tanh (x)$.

It is adopted as the optimum decision function for nonfrequency selective fading channels with the MAI modeled as AWGN in the systems having a large number of users.

\section{Simulation Results}

This section shows the several simulation experiments to test the performance of the proposed algorithms and compare them to each other. The simulation environment is based on the downlink synchronous MC-CDMA system in which each user transmits BPSK information symbols. The propagation channel is assumed to be a frequency selective fading channel with $L=3$ paths and a uniform delay profile. More details of the simulation parameters are given in Table 1. All users are assigned the same power.

The BER versus the threshold of the null zone decision function $\left(c_{n}\right)$ at different SNR values and different number of users for the RZF-PIC algorithm is illustrated in Fig. 4. The best choice threshold point is at $\mathrm{C}_{\mathrm{n}}$ optimal=0.2. Fig. 4 shows that $C_{n}$ optimal is nonsensitive to SNR-changes and to system-load changes.

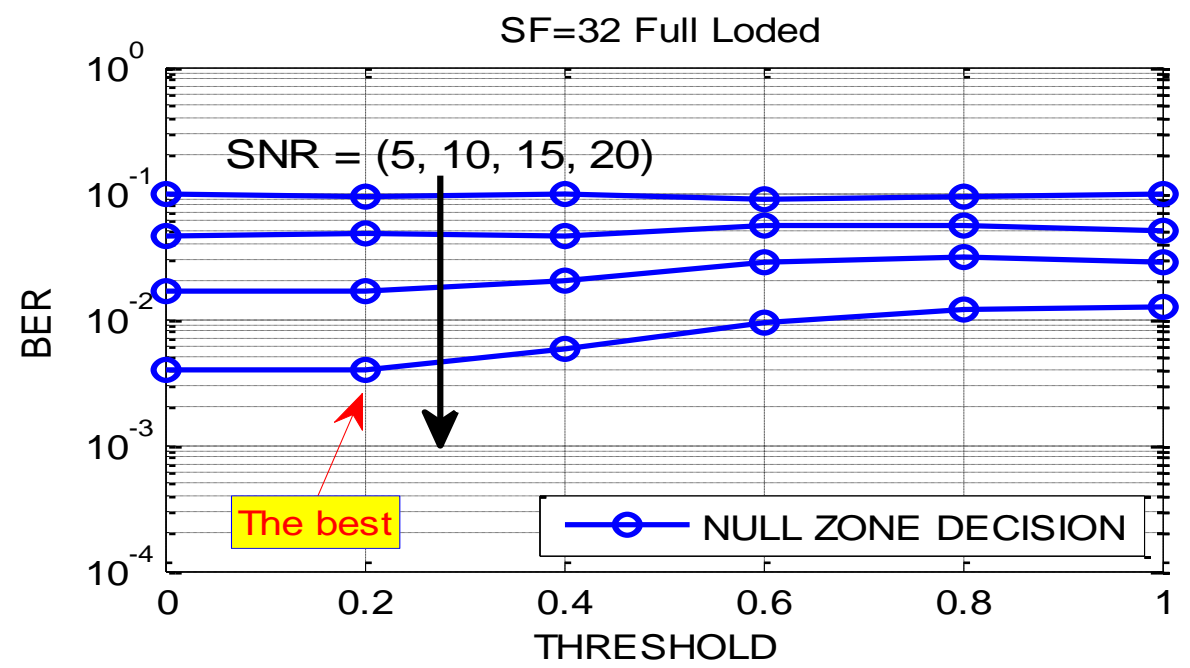

Fig. 4. BER vs. Threshold Parameter ( $\alpha$ ) at different SNRs $K=32$ (full load).

Table 1 Simulation Parameters

\begin{tabular}{l||l|l}
\hline \hline \multirow{4}{*}{ Transmitter } & Modulation & BPSK \\
\cline { 2 - 3 } & Spreading Codes & Walsh-Hadamard codes with spreading factor 32 \\
\cline { 2 - 3 } & FFT points & $P=256$ \\
\cline { 2 - 3 } Channel & Cyclic Prefix & $N_{C P}=32$ \\
\hline \multirow{2}{*}{ Receiver } & Fading & Frequency selective with L=3 \\
\cline { 2 - 3 } & Noise Environment & $A W G N$ \\
\cline { 2 - 3 } & Cqualization & $\begin{array}{l}\text { RAKE }(\text { MRC), } \\
\text { and } R Z \text { F equalizer }\end{array}$ \\
\hline \hline
\end{tabular}


The relation between the regularization parameter $(\alpha)$ and the BER for the RZF-PIC algorithm in the downlink MC-CDMA systems at different SNR values and different load of users is illustrated in Fig. 5. The best choice of $\alpha$ is found at $\alpha=0.01$, and .Thus, the best choice of $\alpha$ is applicable for the rest of experiments.

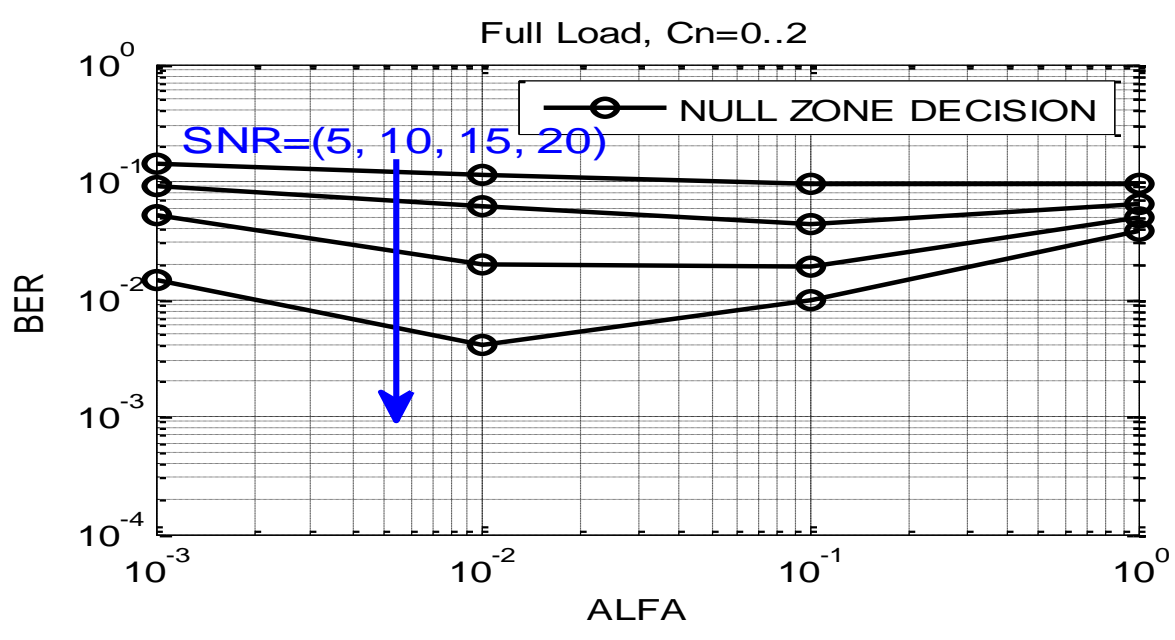

(a)

(b)

Fig. 5. BER vs. Regularization Parameter $(\alpha)$ at different SNRs. $K=32$ (full load).

Figure 6 shows the effect of the tentative decision function on the performance of the RAKEPIC algorithm for $K=32$ (full load). From Fig. 6 the best performance of the RAKE-PIC algorithm can be obtained with the tanh, unit clipper and soft decision functions. On the other hand, the effect of the tentative decision function on the performance of the RZF-PIC algorithm for $K=32$ (full load) is studied and shown in Fig. 7. It is found that the best performance of the RZF-PIC algorithm can be obtained with the hard and null zone decision functions outperform the performance obtained with all other decision functions.

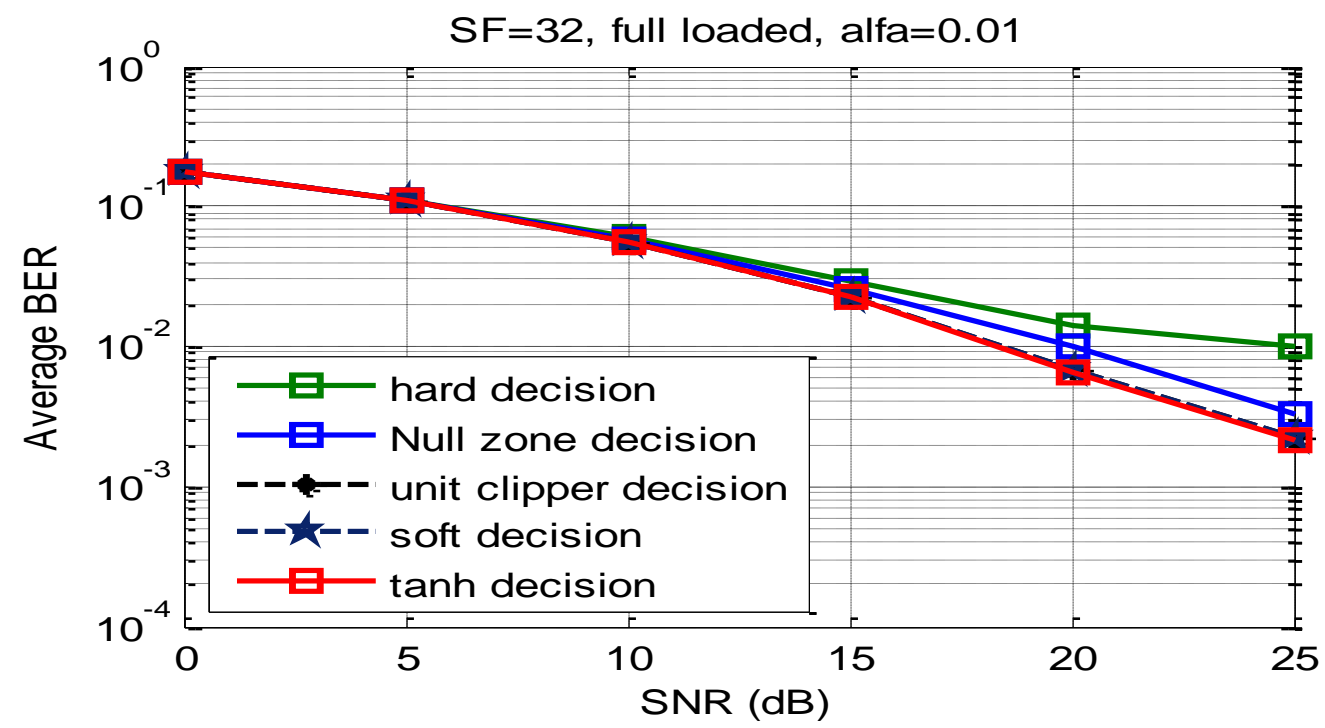

Fig. 6 Performance of different schemes with different decision functions of RAKE-PIC. for $K=32$ (full load). 


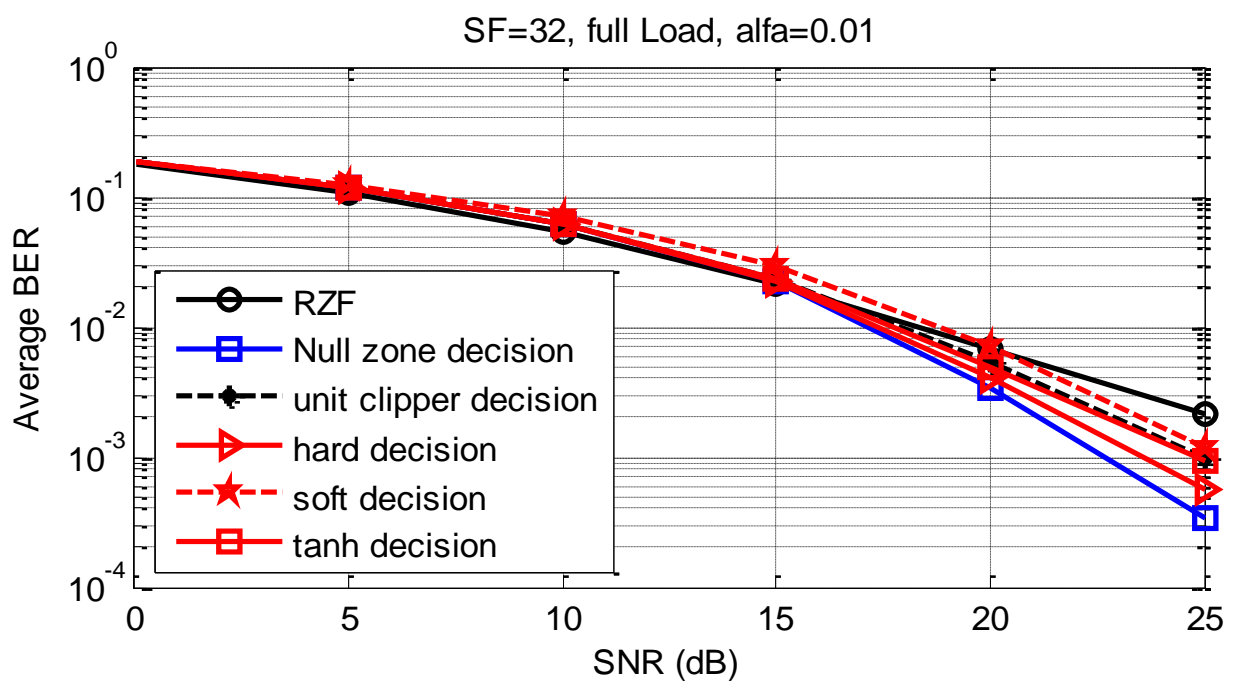

Fig. 7 Performance of different schemes with different decision functions of RZF-PIC, for K=32 (load load)

The comparisons between the three different proposed algorithms for half load and full load of users are introduced in fig. 8 respectively. From the obtained results, it is clear that for low SNR values until $15 \mathrm{~dB}$, the three proposed algorithms have the same performance. At high SNR values, the RZF-PIC algorithm gives the best performance. From fig. 8 the performance of the RAKE-PIC and RZF schemes are the same for the half and full load of users, on the other hand for the half load of users the performance of the RZF-PIC scheme is better than the performance for the full load of users.

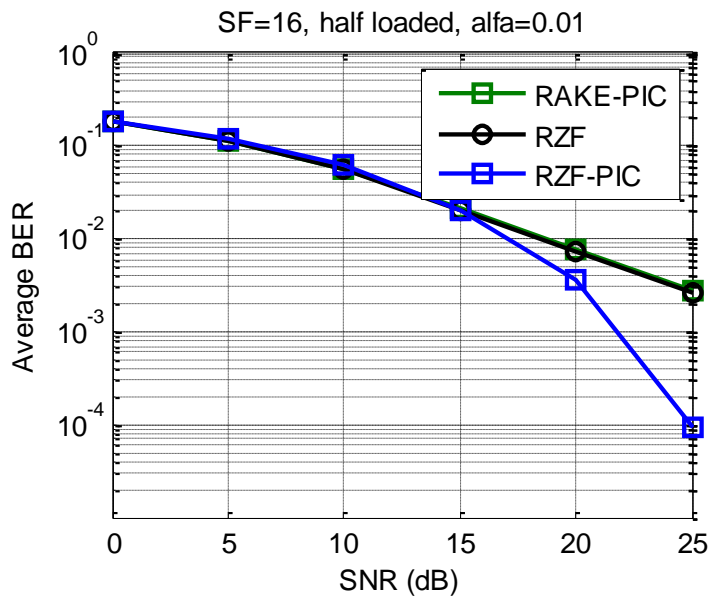

(a)

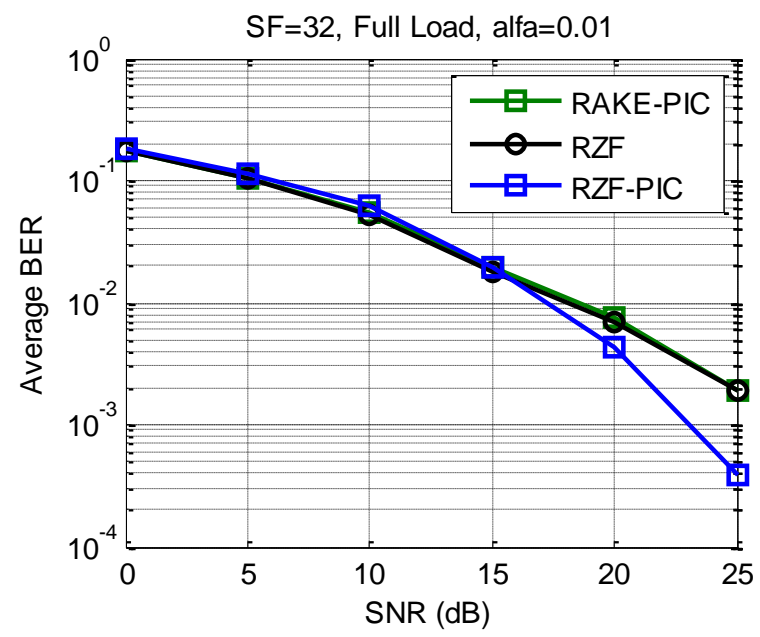

(b)

Fig. 8. BER vs. SNR for different reception schemes. (a) $K=16$ (half load). (b) $K=32$

Figure 9 study and present the effects of user loads on the performance of the tentative decisions of the RZF-PIC algorithm. This figure shows that the performance of the null zone and hard decisions are better than the other decision functions in RZF-PIC algorithm. The BER of all receivers degrades as the number of users increases. 


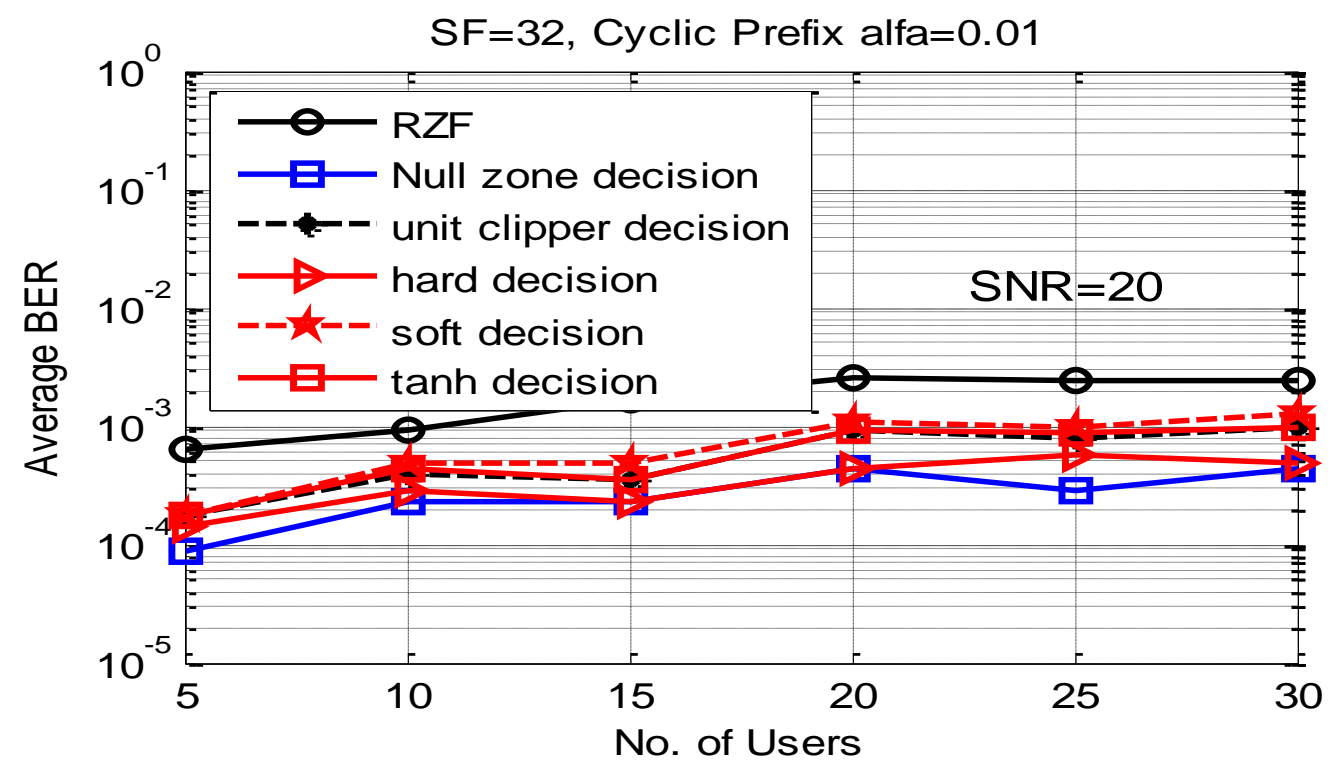

Fig. 9 BER vs. the number of active users for the different tentative decisions for FDE-RZF-PIC algorithm. SNR = $20 \mathrm{~dB}$.

The effects of user loads on the performance of the suggested algorithms are studied and presented in Fig. 10. The BER of all receivers degrades as the number of users increases. This observation may be due to the MAI. The larger of the MAI is occurred during the increasing of the number of users. Even some residual MAI still exists, after interference cancellation. Therefore, the performance loss may be attributed to the residual MAI. From these results in Fig. 10, the performance of the RZF-PIC algorithm degrades a little bit by increasing the number of users, but it is still better than the RAKE-PIC and RZF algorithms.

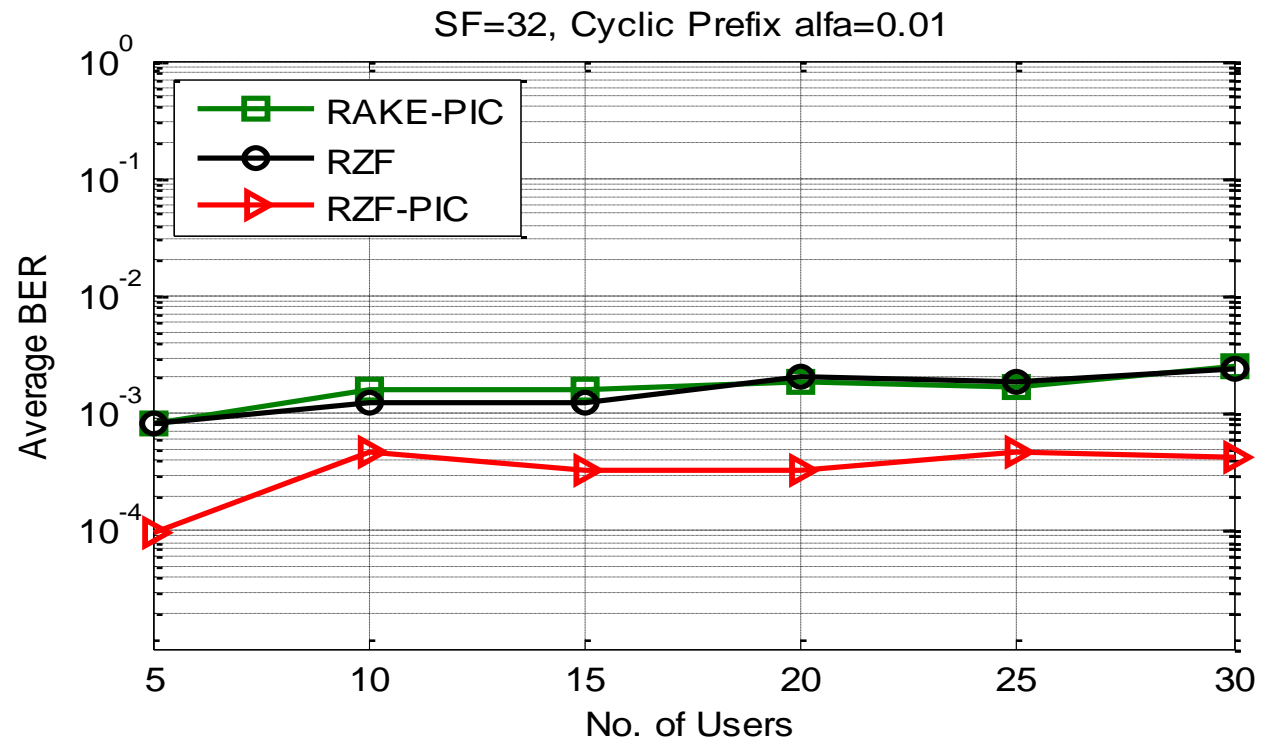

Fig. 10 BER vs. the number of active users for the different reception schemes. $\mathrm{SNR}=20 \mathrm{~dB}$. 


\section{Conclusions}

In this paper, two interference cancellation algorithms have been suggested and studied to down and suppress the interference for downlink MC-CDMA systems. The effects of the tentative decision functions used and the numbers of users on the performance of these algorithms are studied. RZF-PIC gives large performance than RZF and RAKE-PIC. It is found that, the RZF-PIC with hard decision and null zone decision provide better performance than other algorithms at low and heavy loads. This indicates that a reliable communication is possible with the proposed algorithms.

\section{References}

[1] Tsui-TsaiLin, Robust MOE detector for MC-CDMA downlink systems under carrier frequency offset, Signal Processing 89 (2009), pp. 1252-1259.

[2] A.B. Djebbara, K. Abed-Meraimb, A. Djebbaria, Blind channel equalization and carrier frequency offset estimation for MC-CDMA systems using guard interval redundancy and excess codes, Int. J. Electron. Commun. (AEÜ) 63 (2009), pp. 220 225.

[3] Yunlong Cai, Rodrigo C. de Lamare and Didier Le Ruyet, "Transmit Processing Techniques Based on Switched Interleaving and Limited Feedback for Interference Mitigation in Multi-antenna MC-CDMA Systems", IEEE 2011..........

[4] I. Martoyo, T. Wesis, F. Capar, F. Jondral, Low complexity CDMA downlink receiver based on frequency domain equalization, in: Proc. IEEE VTC, Oct. 2003, pp. 987991.

[5] Tingting Liu, Jian-Kang Zhang, Senior Member, IEEE, and Kon Max Wong, Fellow, IEEE, Optimal Precoder Design for Correlated MIMO Communication Systems Using Zero-Forcing Decision Feedback Equalization, IEEE TRANSACTIONS ON SIGNAL PROCESSING, VOL. 57, NO. 9, SEPTEMBER 2009.

[6] I. Martoyo, T. Wesis, F. Capar, and F. Jondral, "Low complexity CDMA downlink receiver based on frequency domain equalization," IEEE VTC., pp. 987-991, Oct. 2003.

[7] F. Petre, G. Lues, L. Deneire, and M. Moonen, "Downlink frequency domain chip equalization for single carrier block transmission DS-CDMA with known symbol padding," in Proc. GLOBCOM, pp. 453-457, Nov. 2002.

[8] S. Tomasin, and N. Benvenuto, "Frequency domain interference cancellation and nonlinear equalization for CDMA systems," IEEE Trans. wireless Commun., Vol. 4, No. 5,pp. 2329-2339, Sept. 2005.

[9] F.S. Al-kamali, M.I. Dessouky, B.M. Sallam, F.E. El-Samie, Parallel interference cancellation and linear equalization for multirate downlink CDMA systems, in: ICCTA 2007, Alexandria, Egypt, 1-3 Sept. 2007, pp. 180-186.

[10] A.L.C. Hui, K.B. Letaief, Multiuser asynchronous DS/CDMA detectors in multipath fading links, IEEE Trans. Commun. 46 (1998), pp. 384-391.

[11] L.B. Nelson, H.V. Poor, Iterative multiuser receivers for CDMA channels: An EMbased approach, IEEE Trans. Commun. 44 (1996), pp. 1700-1710.]

[12] S. Werner, J. Lilleberg, Downlink channel decorrelation in CDMA systems with long codes, in: IEEE 49th VTC, vol. 2, 16-20 May 1999, pp. 1614-1617.

[13] Li Guo and Yih-Fang Huang, Fellow, IEEE,. Interference Suppression for Multiuser Downlink Transmission in Frequency-Selective Fading Channels, IEEE Transactions on Signal Processing, VOL. 56, NO. 9, SEPTEMBER 2008. 
[14] S. Tomasin, N. Benvenuto, Frequency domain interference cancellation and nonlinear equalization for CDMA systems, IEEE Trans. Wireless Commun.4 (5) (2005), pp. 2329-2339.

[15] F.S. Al-kamali, M.I. Dessouky, B.M. Sallam, F.E. El-Samie, Efficient implementation of downlink CDMA equalization using frequency domain approximation, UBICC J. 2 (4) (2007).

[16] F. S. Al-kamali, M. I. Dessouky, B. M. Sallam, and F. E. El-Samie, "Performance of Cyclic Prefix CDMA Systems with Frequency Domain Interference Cancellation," submitted for publication in the digital signal Processing Journal, Elsevier Inc.2009, pp. 2-13. 